\title{
EL DESAFÍO ENTRE LO TEÓRICO Y LO PRÁCTICO: UN RECUENTO DE EXPERIENCIAS DIDÁCTICAS UTILIZANDO HERRAMIENTAS TIC EN EL TALLER DE PRODUCCIÓN EDUCATIVA PARA EL BACHILLERATO EN LA ENSEÑANZA DE LOS ESTUDIOS SOCIALES Y EDUCACIÓN CÍVICA
}

\author{
THE CHALLENGE BETWEEN THE THEORETICAL AND THE PRACTICAL: A \\ COUNTING OF TEACHING EXPERIENCES USING TIC TOOLS IN THE \\ EDUCATIONAL PRODUCTION WORKSHOP FOR THE BACHELOR'S DEGREE IN \\ SOCIAL STUDIES AND CIVIC EDUCATION
}

\author{
Roberto Granados Porras
}

Fecha de recepción: 01/11/2017

Fecha de aceptación: 27/11/2017

\begin{abstract}
Resumen: El siguiente artículo reflexiona sobre la Enseñanza de los Estudios Sociales y la Educación Cívica y su vinculación con el uso de las tecnologías digitales. Además, recopila las experiencias de aprendizaje del Taller de Producción Educativa que se imparte en la carrera y que se considera como un espacio de reflexión e innovación educativa para el futuro profesorado.
\end{abstract}

Palabras clave: Estudios Sociales, Educación Cívica, Taller de Producción Educativa, TIC, innovación educativa.

Abstract: The following article reflects on the Teaching of Social Studies and Civic Education, and its link with the use of digital technologies. In addition, it collects the learning experiences of the Educational Production Workshop that is taught in the career and that is considered as a space for reflection and educational innovation for future teachers.

Keywords: Social Studies, Civic Education, Educational Production Workshop, ICT, Educative Innovation.

\section{Introducción}

La incorporación de las TIC en la actualidad obedece a procesos de innovación educativa que se desarrollaron con mayor preponderancia durante la última década, con el objetivo de buscar una mejora continua en el proceso de enseñanza-aprendizaje. En el caso de los Estudios Sociales y la Educación Cívica, el acercamiento a las tecnologías digitales es pausado y parte del profesorado es escéptico a su aplicación en la mediación pedagógica. Aunque, se parte

\footnotetext{
* Costarricense. Máster en Historia Aplicada por la Universidad Nacional de Costa Rica (UNA) y Licenciado en Estudios Sociales y Educación Cívica por la Universidad Estatal a Distancia, Costa Rica (UNED). Profesor e investigador de la Universidad Nacional de Costa Rica (UNA). Coordinador del Proyecto Gestión de la Calidad BEESEC. Correo electrónico: c.roberth@gmail.com
} 
de la premisa que las tecnologías aplicadas a la educación no es la única vía de mejora, forma parte del trayecto que puede llevar a una innovación educativa.

Por lo tanto, desde el Taller de Producción Educativa se desarrollan diferentes propuestas didácticas con el objetivo de encontrar la motivación del futuro profesorado y que además, puedan crear materiales didácticos funcionales en su futura carrera profesional. Si se llegara a este contexto la innovación educativa sería parte de esta propuesta, tomando en cuenta que dentro de los Estudios Sociales, la Historia se convierte en una disciplina preponderante y se necesitan diferentes técnicas didácticas para mejorar su enseñanza y aprendizaje.

\section{Desarrollo}

\section{Marco teórico}

Las Tecnologías de Información y Comunicación (TIC) Abarca (2014) las define como:

[...] el conjunto de tecnologías que permiten el acceso, producción, tratamiento y comunicación de información, presentada en diferentes formatos de tipo textos, imágenes, audio, vídeo entre otros, supone un cambio en la forma de como las personas adquieren información y se relacionan (p. 33).

De acuerdo con la cita anterior, el profesorado de Estudios Sociales utiliza muchas de ellas que, al pasar de los años, se han hecho más frecuentes como los videos, imágenes y audios. Pero todavía hace falta trabajar en mayor medida en el ámbito de la producción, pues son pocos los materiales directamente elaborados por el colectivo docente. Si se llegara a este contexto, se mejoraría en la calidad de materiales que se emplean en la mediación pedagógica.

A inicios de la década del 2000, se señalaban los cambios tan vertiginosos que desde el ámbito tecnológico experimentaba la sociedad. López \& Schmelkes (2003) propusieron que:

En la sociedad contemporánea, las Tecnologías de la Información y Comunicación (TIC) han tenido un impacto desconcertante que ha producido cambios insospechados semejantes a los que en una época originó la televisión o, aún antes, el teléfono. Sus 
efectos y alcance no sólo se han situado en el terreno de la información y comunicación, sino que lo sobrepasan para llegar a provocar y proponer cambios en la sociedad, la economía, la política y han llegado ya a las instituciones educativas de todos los niveles (p. 203).

Si se hace alusión al contexto actual, estos cambios tecnológicos se aceleraron con mayor ritmo y llegaron prácticamente a todos los rincones del planeta. Por ejemplo, la telefonía celular presentó un crecimiento acelerado y el smartphone se volvió parte de la cotidianidad. Todas estas transformaciones tecnológicas llegaron a los contextos educativos para abrir debates constantes sobre su necesidad o no en los salones de clase; pero estos cambios continuarán y la comunidad docente debe estar anuente a la receptividad de sus consecuencias en los contextos escolares.

Siguiendo con la línea anterior, se retoma el aporte de las tecnologías educativas, que son las que directamente se aplican en este estudio. Primeramente, para conocer mejor el concepto, se inicia con la definición de tecnología que se entiende como: "una de las disciplinas socialmente significativas que, mediante diversas actividades, responde a las demandas individuales o colectivas que presenta la sociedad" (Fallas \& Trejos, 2013, p. 4). De acuerdo con 3 lo anterior, se utiliza la postura de Ramas et al. (2015) que cita a Frisch (1960) al resaltar la capacidad del ser humano de contralar su entorno con herramientas y definiéndolo como Homo faber, el cualtraducido al castellano sería "hombre tecnológico" puesto que sus habilidades le permiten "emplear técnicas para resolver problemas prácticos" (p.17).

Las tecnologías llegaron al campo de la educación con las computadoras, internet, la telefonía, las comunicaciones satelitales, las aplicaciones multimedia y la realidad virtual, los cuales proporcionan nuevos canales de información y comunicación, principalmente vinculados a dispositivos informáticos. Aunque este desarrollo tecnológico se relaciona con un proceso de mercado y globalización, desde el ámbito educativo, se busca la aplicación en el aula y que el estudiantado pueda desarrollar habilidades que lo lleven a responder problemas prácticos de la vida cotidiana y participar en una sociedad con altas competencias tecnológicas. En esta línea, Ramas et al. (2015) proponen: "La nueva organización informacional exige a los individuos a 
tomar conciencia de sus potencialidades y delimitar los alcances tecnológicos a fin de asumir las transformaciones sociales y sus efectos a futuro" (pp. 18-19).

Pero existe un punto clave dentro de este paradigma de innovación y es la formación del profesorado. Como se sabe, la instrucción del colectivo docente no termina en las aulas universitarias, individualmente deben gestarse procesos de actualización que motiven el camino de la innovación. La formación del profesorado se entiende como la capacitación, participación en cursos, posgrados, seminarios y diferentes actividades académicas e institucionales con el fin de mejorar su labor docente. En esta línea, Serrano \& Pontes (2016) argumentan que el colectivo docente se constituye como uno de los principales agentes que puede hacer posible el cambio educativo, ajustándose a un perfil profesional que le permita responder de forma adecuada a los nuevos retos y demandas que están surgiendo en la sociedad del conocimiento e información.

Según Serrano \& Pontes (2016) la formación del profesorado está ligada a un concepto que acuñó Erickson en 1980 que habla sobre la Identidad Profesional Docente (IPD) y que se compone de dos elementos claves como: "una dimensión personal, íntima y peculiar que hace al sujeto diferente de los y las demás, y una dimensión social, compartida y en constante interacción con «otros y otras»" (p. 34). Es decir, el profesorado individualmente desarrollará su identidad profesional que alimentará con el paso de los años en los diferentes contextos educativos donde desarrollará su práctica cotidiana.

Ahora bien, como se habló anteriormente que, aunque, los Estudios Sociales y la Educación Cívica comprenden diferentes disciplinas, uno de los ejes principales de esta investigación es apuntar al mejoramiento de la enseñanza de la Historia. Desde esta asignatura, enseñar Historia es uno de los retos más significativos, pues el estudiantado considera su aprendizaje como aburrido y mecánico. Esta percepción o realidad no es culpa del estudiantado por sí mismo, sino del profesorado. Al respecto Taracena (2015) menciona:

En la actualidad no resulta extraño escuchar que la historia es aburrida. Este es un reclamo generalizado que ha alcanzado atributo de sentido común al señalar la enseñanza de la historia como una materia dedicada a memorizar datos (p. 11). 
Es decir, la etiqueta que lleva la enseñanza de la Historia puede afectar metodológicamente su función social, pues existe una negación y apatía en todos los niveles de enseñanza. Siguiendo con la propuesta de Taracena (2015), la "historia aburrida" se ha convertido en un estigma y lo más problemático de esta situación, es que se niega-desaprueba su utilidad para enfrentar problemas de la vida cotidiana. Tomando en cuenta que, en otros contextos de aprendizaje como el universitario, la Historia se relaciona como un conocimiento erudito al que pocos pueden alcanzar, siendo esto una afirmación que la presente investigación refuta.

Por lo tanto, todas las problemáticas anteriores llevan a una nueva visión de la enseñanza de la Historia. Se busca una renovación de los contenidos y sobre todo de la aplicabilidad de lo que se enseña en las aulas, pues la utilidad del currículo en Historia es prácticamente efímera, en el sentido que las actividades de mediación del profesorado, en la mayoría de los casos, no incitan al estímulo de competencias necesarias en la actualidad.

Como consecuencia de lo anterior, se propone una relación entre la enseñanza de la Historia y el uso de la tecnología, para mejorar la metodología con que se tratan los temas históricos en los contextos de enseñanza aprendizaje. Collazo \& de Ranalletti (2015) argumentan que: "[...] lo más significativo en el terreno de las ciencias sociales se puede señalar en cuanto a la participación del estudiante en la creación del conocimiento" (p. 8). Metodológicamente se parte de esta idea, en el sentido que la enseñanza de la Historia se debe hacer vivencial y el estudiantado debe participar en la creación del conocimiento. Para ello, los enfoques de las TIC colaboran significativamente en este abordaje y se pueden emplear en las diferentes actividades de clase.

En la actualidad, muchos de los aprendizajes son mediados por la tecnología, es por eso que la enseñanza de la Historia presenta amplias ventajas al contar con diversas herramientas que colaboran en la mediación docente. Basado en lo anterior, Collazo \& de Ranalletti (2015), citando a Meyer (2001), señalan que los aprendizajes multimedia que se caracterizan por "seleccionar o diseñar herramientas pedagógicas con empleo de recursos multimedia y digitales" (p. 10). 
Para explicar la concepción actual de estos aprendizajes Collazo \& de Ranalletti (2015) proponen una tipología que se caracteriza por:

La atención dividida: el material didáctico no debe requerir al alumno dividir su atención en múltiples fuentes referidas a una misma información. La modalidad: la información verbal debe ser presentada de preferencia auditivamente puesto que el alumno la comprende mejor que cuando se presenta visualmente (texto). La contigüidad espacial: el material visual y el texto deben estar integrados, en lugar de exponerse por separado. $L a$ contigüidad temporal: los materiales verbal y visual han de ser presentados de manera simultánea o próxima. La coherencia: se debe excluir cualquier material irrelevante al contenido que se quiere enseñar en la presentación multimedia (sonidos, música, imágenes, etc.) (pp. 10-11).

En la planificación de clases basadas en este tipo de aprendizajes debe coexistir una secuencia lógica que supervise el adecuado desarrollo de las lecciones. Además, el profesorado debe poseer un papel destacado que se caracterice por un rol activo y una mediación que incite al estudiantado a plantearse retos y soluciones a los problemas presentes. Al respecto, Taracena (2015) expresa que el objetivo de la tecnología consiste en su utilidad, teniendo en cuenta que impulsan nuevas habilidades y pensamientos flexibles. Dejar la metodología convencional de la enseñanza de la Historia para repercutir en "la acción social, en los cambios culturales, en los imaginarios y en las esperanzas colectivas actuales" (p. 16).

Pero, dentro de la propuesta a desarrollar, para que exista un buen engranaje debe existir una adecuada mediación pedagógica que se considera como el acompañamiento que lleva a cabo el profesorado con el colectivo estudiantil, el cual se caracteriza por ser un guía que estimule los aprendizajes significativos con actividades de clase que se ajusten a la realidad de los y las estudiantes. Por lo tanto, el mediador es la persona que al relacionarse con otra u otras, según Ferreiro \& Vizoso (2008) propicia el paso del sujeto que aprende de "un estado inicial de no saber, poder o ser, a otro cualitativamente superior de saber, saber hacer y, lo que es más importante, ser" (p.76). El mediador, por lo tanto, debe propiciar el aprendizaje, y proponer desarrollo de potencialidades y corregir funciones cognitivas deficientes; mueve, en términos 
vigotskianos, al sujeto que aprende en su zona de desarrollo potencial (Ferreiro \& Vizoso, 2008, p.76).

Ferreiro \& Vizoso (2008), citando el enfoque vigotskiano, proponen que el aprendizaje está condicionado por la presencia de otra persona más diestra y conocedora, de la interacción social entre esa persona y el otro, de la negociación semiótica que se logra en el proceso de comunicación entre ellos y la actuación que le brinda ayuda al que aprende en los márgenes de su zona de desarrollo potencial. De acuerdo con esta propuesta, el docente debe utilizar su conocimiento acumulado en el transcurso de los años para generar situaciones de aprendizaje que sean significativas para el estudiantado, tomando en cuenta todas las diferencias y necesidades del estudiantado.

También, lo anterior se traduce en un concepto que Vigotsky llamó "la necesidad de la ayuda del otro para aprender". Este se debe complementar con distintos factores para que sea efectiva. En primera instancia, se debe tener en cuenta la relación entre lo actual y lo potencial, es decir, lo que puede hacer por sí solo el sujeto que aprende y lo que podría llegar a realizar con la ayuda que se le brinda. Solo así el aprendizaje deja de ser una simple apropiación y se convierte en algo que provoca desarrollo cognitivo (Ferreiro \& Vizoso, 2008, p.78).

Es así como, el proceso de mediación se caracteriza por la interacción de dos o más sujetos interesados por un objetivo específico, en la que al menos uno juega el papel de mediador. Por lo tanto, las actividades que propone el docente son las herramientas mediadoras que pueden llevar al estudiantado a obtener un nuevo aprendizaje. Para nuestra investigación, el empleo de los recursos tecnológicos no media por sí misma. El que media es el profesor que emplea la tecnología con una intención para "mover al alumno en su zona de desarrollo potencial, ya bien en lo individual, ya en lo grupal" (Ferreiro \& Vizoso, 2008, p.80).

En los Estudios Sociales y, específicamente en el desarrollo de los contenidos de Historia, existen problemas de mediación. El profesorado, en muchos casos, se ha convertido en un trasmisor vertical de contenidos y como consecuencia las actividades de clase no se ajustan a las necesidades educativas del colectivo discente. Por eso, desde la mediación pedagógica en Historia, se necesita una renovación y un compromiso del profesorado de Estudios Sociales, para llegar a una práctica pedagógica adecuada. 
Por lo tanto, la propuesta central desde el Taller de Producción Educativa es la búsqueda de los aprendizajes significativos que se presentan como un eje medular de la presente investigación. Es así como se plantea el aporte teórico del constructivismo que argumenta “[...] los individuos viven en el mundo de sus propias experiencias personales y subjetivas. Siendo el individuo quien impone significado sobre el mundo, en vez del mundo imponerle su significado, construyendo una visión personal de la realidad" (Karagiorgi \& Symeou, 2005, citado por Cenich \& Santos, 2010, p. 8). Por lo anterior, para generar aprendizajes significativos en Estudios Sociales, el profesorado no debe fungir como un transmisor de contenidos sino como un mediador que promueva un intercambio y debate que propicie el aprendizaje como un fenómeno de construcción personal antes de un proceso de transmisión.

Desde la propuesta del constructivismo social, Cenich \& Santos (2010), citando a Vygotsky (1930 y 1978), argumentan que la cultura y el contexto son importantes en la formación y adquisición del conocimiento. En este paradigma, el aprendizaje no es un proceso interno, sino un constructo social mediado por el lenguaje, donde el contexto constituye el centro del aprendizaje. Asimismo, el aprendizaje consiste en la internalización progresiva de los instrumentos mediadores en un proceso de enseñanza y aprendizaje, donde se considera al que aprende, al que enseña y la relación social entre ellos.

Entonces, de acuerdo con la propuesta anterior, el profesorado mantiene un rol de guía que debe posibilitar en el estudiantado habilidades con un grado de complejidad mayor conforme avancen los contenidos de estudios, para así estimular competencias que conlleven a los aprendizajes significativos. Cenich \& Santos (2010) relacionan esta concepción con la zona de desarrollo próximo (ZDP) de Vygotsky propuesta en el constructivismo social definida como:

[...] la distancia entre el nivel real de desarrollo, determinado por la capacidad de resolver independientemente un problema, y el nivel de desarrollo potencial, determinado a través de la resolución de un problema bajo la guía de un adulto o en colaboración con otro compañero más capaz (p. 8).

El aprendizaje intencional se apoya frecuentemente en las actividades de mediación pedagógica, propuestas por el profesorado para proporcionar contextos que se caracterizasen por 
ser nuevas experiencias que estimulen actividades significativos. Por lo anterior, el profesorado se caracteriza por promover los aprendizajes que parten del contexto cultural y de los aprendizajes previos, determinando la construcción de un nuevo conocimiento. De acuerdo con la teoría del aprendizaje significativo de David Ausubel (1976), citado por Cenich \& Santos (2010), menciona que se aprende a partir de aquello que se conoce, de esta manera para promover el aprendizaje significativo cobra relevancia los conocimientos previos del estudiantado.

Por último, pero a la vez se convierte en uno de los ejes centrales de esta propuesta teórica se encuentra el concepto de innovación educativa que contempla aspectos como: tecnología, didáctica y pedagogía. Para López \& Heredia (2017)

Una innovación educativa implica la implementación de un cambio significativo en el proceso de enseñanza-aprendizaje, de los materiales empleados para el mismo, de los métodos de entrega de las sesiones, de los contenidos o en los contextos que implican la enseñanza (p.18)

Según la propuesta de las autoras, la innovación implica una diferencia que se debe 9 relacionar con la calidad y lo novedoso de aquellos elementos que se someten a una mejora continua en busca de llegar a entregar un aporte sustancial al proceso de enseñanza-aprendizaje. Además, proponen una escala que se caracteriza por la innovación disruptiva, la cual tiene la capacidad y potencial desconocidos para afectar a todo y a todos los que actúan en el contexto educativo; innovación revolucionaria que muestra la aplicación de un nuevo paradigma y se revela como un cambio fundamental en el proceso de enseñanza-aprendizaje y un cambio significativo de las prácticas existentes; innovación incremental que se construye con base en los componentes de una estructura ya existente, dentro de una arquitectura o diseño establecido y la innovación continua, que promueve cambios que solamente afectan parcial o limitadamente a los procesos o elementos del contexto educativo, o que proponen su mejora como eficiencia de operación, entrega o procedimiento (López \& Heredia, 2017, p.18).

\section{Planteamiento del problema}

Revista Perspectivas: Estudios Sociales y Educación Cívica - No. 15

ISSN-L: 2215-4728 • julio-diciembre $2017 \bullet$ pp. 1-21

DOI: http://dx.doi.org/10.15359/rp.15.3 
De acuerdo con la propuesta teórica y tomando como base los procesos de innovación educativa y la búsqueda de una formación que estimule procesos de aprendizaje que sean significativos para el nuevo profesorado de Estudios Sociales y Educación Cívica y, que sean alicientes para buscar un cambio educativo en los diversos entornos escolares se planteó la siguiente propuesta que busca responder a: ¿Cómo el Taller de Producción Educativa impartido en el Bachillerato de Estudios Sociales y Educación Cívica se convierte en un espacio de innovación educativa para el futuro profesorado?

\section{Método}

En la presente investigación, se utilizó paradigma naturalista, que también se conoce como naturalista-humanista o interpretativo. Según Barrantes (2014) el interés de este paradigma se centra "en los significados de las acciones humanas y de la vida social" (p. 82). Derivado de lo anterior, la investigación se desarrolló por medio de un enfoque cualitativo, que en palabras de Hernández, Fernández \& Baptista (2010) se define como: "la recolección de datos sin medición numérica para descubrir o afinar preguntas de investigación en el proceso de investigación" (p. 7).

Dentro del tipo de investigación y, derivado del enfoque cualitativo, el trabajo se basó en las modalidades de estudio de la investigación-acción y la fenomenología. Según Campos \& Madriz (2015) este tipo de investigación puede ser definida de muchas maneras, pero "su característica principal es el hecho de que forma parte de un proceso de transformación de la realidad social por acciones propias de los protagonistas inscritos a ella" (p. 6).

En ejecución, el Taller de Producción Educativa es un curso presencial que destina la mayor parte de la clase a la práctica con herramientas tecnológicas y a la promoción del trabajo colaborativo entre el estudiantado. Desde la teoría, se profundiza con la lectura de textos sobre el papel de las TIC en la actualidad y su relación con las Ciencias Sociales. Por lo tanto, el eje central del taller es estimular al nuevo colectivo docente para que pueda generar una transformación pedagógica que permita la creación de espacios de innovación educativa.

Por su parte, el estudiantado participante del taller pertenece al III nivel de la carrera. En el segundo semestre del 2016 se contó con 15 estudiantes matriculados y en el 2017 con 20. Por 
lo tanto, a nivel curricular se pretende que el estudiantado pueda analizar los nuevos enfoques de enseñanza-aprendizaje, y con el empleo de recursos tecnológicos generar espacios de innovación en la enseñanza de los Estudios Sociales y la Educación Cívica. NO se dice cómo está compuesto el taller.

Desde esta óptica anterior, el estudiantado contó con una estrategia de evaluación dinámica y versátil basada en la producción colectiva. Cada una de las actividades propuestas en el curso contó con un espacio de socialización, para fortalecer el aprendizaje en equipo y visualizar cada una de las deficiencias que pudieran corregirse dentro de la propuesta curricular. En esta dinámica de curso el proceso de enseñanza-aprendizaje es horizontal, puesto que en la actualidad el estudiantado cuenta con competencias tecnológicas y teóricas que pueden hacer del desarrollo académico una experiencia innovadora.

\section{Resultados}

Posterior a la conclusión del taller, fue importante analizar la concepción del estudiantado sobre las posibilidades de la tecnología educativa como espacio de renovación didáctica en la enseñanza de los Estudios Sociales y la Educación Cívica. Al consultar la posición del colectivo estudiantil sobre la temática, las respuestas en su mayoría fueron positivas. En primera instancia, se argumenta sobre las amplias "opciones metodológicas que presentan dichas herramientas" (Entrevista al estudiantado \#2, ítem 2, 1 noviembre 2017). En este sentido, el argumento se relaciona con la posibilidad de desarrollar diferentes actividades didácticas, donde la incorporación de la tecnología impulse una transformación sustancial en el proceso de enseñanza-aprendizaje.

Otro de los entrevistados argumentó que la incorporación de dichas herramientas en la mediación pedagógica de Estudios Sociales y la Educación Cívica es un "medio para visualizar elementos fundamentales y que para dicha asignatura posibilita una variedad de escenarios para la enseñanza de sus contenidos" (Entrevista al estudiantado \#2, ítem 2, 1 noviembre 2017). De acuerdo con lo anterior, se puede concluir que el futuro profesorado tiene claro las posibilidades de transformación que presenta la tecnología en los contextos educativos. Aunque, está claro que 
la incorporación de las TIC depende de las posibilidades y proyección que los centros educativos le otorguen a esta temática.

En la misma línea, otro participante del curso expone la importancia de las tecnologías digitales y su incorporación en los contextos de aula al mencionar que:

[...] Utilización de las TICs como herramientas para el desarrollo de una mediación más activa, innovadora y ligada al uso de la tecnología que está siendo parte de la vida de las

y los estudiantes costarricenses, independientemente el contexto en que se ubican (Entrevista al estudiantado \#2, ítem 2, 1 noviembre 2017).

De la cita anterior se rescata que, sin importar el contexto, el acceso tecnológico se hace cada vez más frecuente en las poblaciones educativas de todos los rangos etarios. Por lo tanto, el profesorado debe hacer lectura de los aportes que el acceso tecnológico lleva a los espacios de aula. Por consiguiente, se debe aprovechar el nivel cognitivo del estudiantado en la temática, para desarrollar actividades didácticas centradas en las necesidades del colectivo estudiantil.

Ahora bien, es importante mencionar que la mayoría de problemáticas que enfrenta el profesorado de Estudios Sociales y Educación Cívica no se pueden corregir empleando la tecnología como único espacio de innovación educativa. La mayoría de ellas son situaciones estructurales o propias del colectivo docente. Dentro de la propuesta de investigación, una de las interrogantes fue obtener las apreciaciones del futuro profesorado sobre las situaciones que más afectan a sus colegas en ejercicio, y se argumentó que en mayor medida el conformismo, la negativa a la actualización docente y la sobrecarga de trabajo son las mayores problemáticas. Para ejemplificar lo anterior, veamos la figura 1:

\section{Figura 1 \\ Problemáticas del profesorado en ejercicio según apreciaciones del estudiantado participante en el Taller de Producción Educativa, II ciclo 2017}

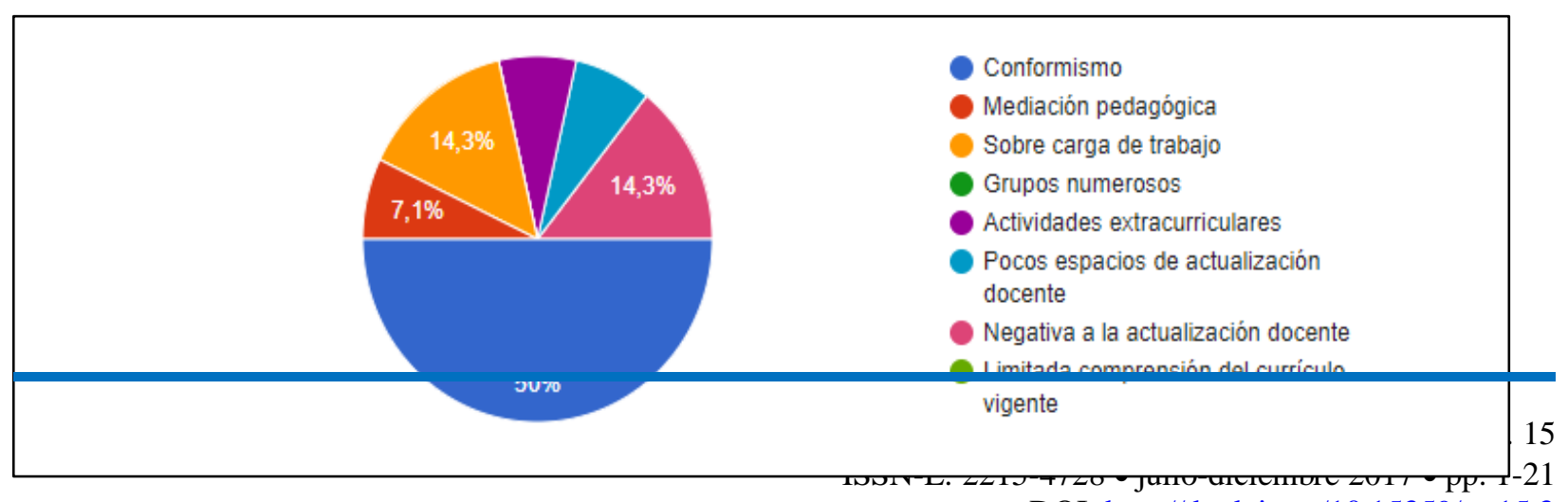


Fuente: Elaboración propia. Entrevista aplicada al estudiantado \#1, ítem 3, 24 de setiembre 2017.

Además, existen otros elementos que se presentan en la figura anterior, que según las apreciaciones, repercuten en los contextos de aula. Uno de ellos es la actualización docente, que parte del profesorado deja de lado y no la visualiza como un espacio de innovación educativa. Pero también, existen otros elementos como la sobrecarga de actividades extracurriculares que puede interferir en un adecuado desarrollo de la actividad docente es Estudios Sociales y Educación Cívica. Ahora bien, es responsabilidad del profesorado en ejercicio visualizar sus propios espacios de actualización, con el objetivo de influir positivamente en la calidad de la educación.

Ahora bien, cuando se preguntó por qué se señaló las opciones presentes en la figura anterior las respuestas fueron variadas. Por ejemplo, cuando se argumenta sobre el conformismo se alude lo siguiente:

[...] entran en una zona de confort, donde la actualización y compromiso de mejorar en los procesos de enseñanza-aprendizaje, se ven reducidos a una rutina repetida por años, en un principio por los docentes que formaron a los jóvenes docentes, que terminan repitiendo las formas en las que fueron enseñados (Entrevista al estudiantado \#1, ítem 4, 24 de setiembre 2017).

La cita anterior menciona un elemento clave con relación a la formación de futuros docentes, pues corren el riesgo de convertirse en transmisores del formato de lecciones que recibieron en las universidades. Principalmente, una clase magistral donde la construcción del aprendizaje se limita a la escucha y la toma de notas. Situación que la refuerza uno de los entrevistados al mencionar que: "Los docentes ya no buscan como salir de sus clases magistrales. En vez de innovar y ser más docentes, las evaluaciones son las mismas de hace 30 años [...]" (Entrevista al estudiantado \#1, ítem 4, 24 de setiembre 2017). Por lo tanto, la lejanía de esta 
metodología didáctica puede favorecer la construcción de aprendizajes significativos en la enseñanza de los Estudios Sociales y Educación Cívica.

Si se toma en cuenta que la innovación educativa “[...] implica la implementación de un cambio significativo en el proceso de enseñanza-aprendizaje [...] (López \& Heredia, 2017, p. 18), se le preguntó a los participantes del Taller si las problemáticas descritas en los párrafos anteriores podrían solucionarse con un proyecto de esta naturaleza y las respuestas fueron favorables, pues un $85.7 \%$ mencionó que un proyecto de este tipo podrían favorecer algunas de las situaciones que se presentan en ésta área como se muestra en la figura 2:

Figura 2

Opinión por parte del estudiantado sobre la aplicación de proyectos de innovación educativa como solución a las problemáticas que enfrenta el profesorado de Estudios Sociales y Educación Cívica

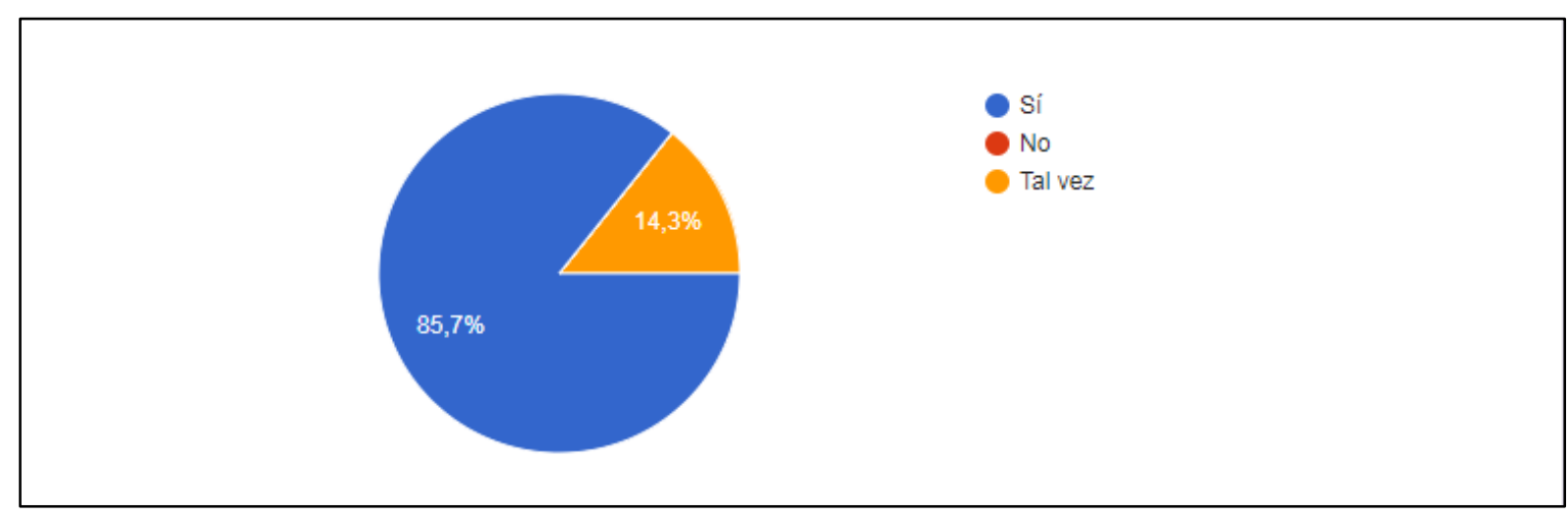

Fuente: Elaboración propia. Entrevista aplicada al estudiantado \#1, ítem 5, 24 de setiembre 2017.

Según algunos participantes del taller, la innovación educativa podría "desarrollar destrezas y habilidades en beneficio de un aprendizaje integral" (Entrevista al estudiantado \#2, ítem 4, 1 de noviembre de 2017). Como se planteó en el referente teórico, dicha innovación podría ser aliciente para solucionar algunas de las problemáticas que se presentan en el sistema educativo, tomando en cuenta que sus propuestas no necesariamente implican el empleo de la tecnología, pues busca cambios transformadores que sean disruptivos, revolucionarios, continuos 
e incrementales. Para el colectivo estudiantil la innovación debe ser "[...] un proceso que genera cambios en los objetivos planteados, con el fin de alejarse de formas tradicionales de enseñanza e incorporar tendencias más actualizadas, con el fin de que el aprendizaje sea significativo" (Entrevista al estudiantado \#2, ítem 4, 1 de noviembre de 2017).

En relación con lo anterior, el Taller de Producción Educativa busca ser un espacio de innovación desde su planteamiento inicial y desde la puesta en escena del curso. Por lo tanto, el trabajo colaborativo fortalece habilidades propias de la carrera que requiere el futuro profesorado. Es así como la incorporación gradual de herramientas TIC promueve la elaboración de materiales didácticos y visualiza posibles espacios donde aplique la mediación tecnológica. Por ejemplo, en el taller impartido en el II ciclo del 2016, se inició con la elaboración de una revista digital que presentó dos funciones: la primera de presentación y la segunda de argumentación. Desde el elemento argumentativo, se enfatizó como podría usarse esta herramienta en un tema de enseñanza de la geografía y se emplearon herramientas como issu, Calaméo y joomang. ${ }^{1}$ La actividad buscó fortalecer el elemento visual que en la disciplina es uno de los factores didácticos que se emplean con mayor frecuencia. Por su parte, en el taller del 2017, el componente visual se complementó con la técnica del Pecha Kucha que promueve la síntesis con la técnica 20X20, 20 filminas en 20 segundos cada una. ${ }^{2}$

Pero además, el empleo del elemento visual se hace necesario en esta disciplina. En búsqueda de una escucha activa y el empleo de la imaginación de quién es el receptor, se elaboró en el taller una propuesta didáctica con audios, para lo cual se utilizó la plataforma ivoox.com. Esta aplicación permite utilizar el repositorio de la plataforma y subir podcast de los usuarios. El empleo de la herramienta propició la búsqueda de nuevos espacios de aprendizaje, tanto el taller del 2016 como en el $2017 .^{3}$

Dentro de la mediación virtual, una de las técnicas más utilizadas son los foros, los cuales permiten sintetizar sobre un tema en específico, generar conclusiones y aportes colaborativos. Dentro del taller, esta herramienta se emplea con frecuencia, pero se prioriza en las discusión de

\footnotetext{
${ }^{1}$ En el siguiente enlace se presenta una de las revistas que se elaboró en el taller: https://issuu.com/rebeckanavarro/docs/tarea_1_curso_taller.docx_3a8ce7ab0f0fab.

${ }^{2}$ Para ampliar sobre la técnica y utilizarse en la mediación pedagógica se recomienda visitar el siguiente sitio: http://www.pechakucha.org.

3 Escuchar: https://mx.ivoox.com/es/12494762.
} 
aspectos relevantes sobre la disciplina y los aportes que la tecnología pueden entregar a ésta. Pero es importante mencionar que no se debe abusar de la herramienta, pues existe una alta probabilidad que desde la mediación universitaria suceda.

Además, a modo de trabajo independiente en los dos talleres se elaboraron tareas de enfoque colaborativo. En el 2016 se desarrolló un mapa conceptual con la herramienta CmapTools y un video sobre una temática histórico. ${ }^{4}$ Con los mapas conceptuales se pretende que el futuro profesorado desarrolle un repositorio personal y lo comparta con su estudiantado. $\mathrm{Al}$ igual con los videos, donde es recomendable que se entre en contacto con las herramientas de edición y producción audio visual. En el taller del 2017, para la edición de las tareas se contó con la herramienta padlet, que es una pizarra interactiva y se puede editar de forma colaborativa. En el trabajo se discutió sobre los nuevos escenarios de la enseñanza de la Historia, pero a la vez, se promovió el uso de pizarras digitales como las que presenta la herramienta. ${ }^{5}$

Para el montaje de asignaciones escolares se promovió el uso de afiches, dado que este tipo de tareas no se presentan visualmente atractivas y dicha técnica puede colaborar en estas actividades. Las herramientas propuestas fueron Mural.ly, Smore y Glogster. ${ }^{6}$ De igual forma, tanto el trabajo cotidiano como extraclase se pueden combinar con el enfoque de aprendizaje invertido, donde el estudiantado previamente ejecuta algunas asignaciones y en la clase se discuten elementos centrales de la temática.

Por último, aunque se elaboraron comics, collage digitales, WebQuest y herramientas para presentaciones en línea, se destaca el desarrollo del PLE - personal learning environmentque se concretó por medio de la elaboración de un blog-portafolio, una página web y un eportafolio. En el taller del 2016, en el blog-portafolio se escribió una reflexión semanal sobre los trabajos que se desarrollaron en el curso, lo que permitió compartir de una forma interactiva de

\footnotetext{
${ }^{4}$ Ver los siguientes videos elaboradores respectivamente en el taller del 2016 y 2017 : https://www.youtube.com/watch?v=JdG5M-HrJMs\&feature=youtu.be, https://www.youtube.com/watch?time_continue=46\&v=8ZkHvVQvJ10.

5 Para analizar el empleo del padlet acceder a: https://padlet.com/andf3n/d1lzj4sw9kc1, https://padlet.com/andresseg15/d895wggku34o, https://padlet.com/josma9424/egg3tk7zlmwh.

${ }^{6}$ Ver algunos de los murales en: https://www.smore.com/91zj6-trabajo-cotidiano, https://www.smore.com/5fz4k.
} 
las actividades; misma propuesta que se utilizó en el 2017 con el e-portafolio. ${ }^{7}$ Por su parte, la página web se elaboró en el 2016 y la propuesta se basó en desarrollar una actividad de innovación educativa para la enseñanza de los Estudios Sociales o Educación Cívica, por lo que parte del estudiantado se inclinó por el desarrollo de una unidad didáctica que se ajustó a los requerimientos de proyectos en innovación educativa. ${ }^{8}$

El objetivo principal de taller es convertirse en un espacio de innovación educativa en la enseñanza de los Estudios Sociales y Educación Cívica, para lo cual fue necesario conocer la opinión del estudiantado sobre las temáticas desarrollada en el curso. Por ejemplo, una argumentación afirmó que el taller: "permitió espacios para poner en perspectiva los contenidos educativos a través de herramientas que crearon un nuevo medio para la construcción de escenarios educativos para la Enseñanza de los Estudios Sociales y la Educación Cívica" (Entrevista al estudiantado \#2, ítem 7, 1 de noviembre de 2017). De acuerdo con lo anterior, cuando el futuro estudiantado visualiza las oportunidades de cambio que presenta la tecnología educativa, las posibilidades de un impacto positivo en los contextos de aula aumentan. Principalmente, por la generación de nuevas estrategias didácticas que se ejecutaran y promueven un espacio de cambio.

Otro de los entrevistados argumenta que el taller si fue un espacio de innovación educativa porque se "desarrollaba mucho la parte creativa del estudiante" (Entrevista al estudiantado \#2, ítem 7, 1 de noviembre de 2017). Lo importante de lo anterior versa en la posibilidad que el colectivo participante se identifique con elementos creativos que luego pueda llevar a la práctica. La propuesta didáctica del curso no sería creativa, si el mismo estudiantado no se identifica con la dinámica de clase y las oportunidades que las herramientas TIC ofrecen a la educación, y en especial al campo disciplinar.

Para finalizar, se le preguntó al estudiantado si la carrera de Estudios Sociales y Educación Cívica debería llevar más cursos de tecnología educativa y un 83,3\% opinó que sí, ante $16,7 \%$ que no (Entrevista al estudiantado \#2, ítem 7, 1 de noviembre de 2017). Lo anterior

7 Como ejemplos de los entornos PLE acceder a: http://alenxandrialibrary.blogspot.com, https://medium.com/@andrsarayavargas/aprobar-o-aprender-d6c00bb1c5ea, https://medium.com/@jose1996bp/aprendizaje-sin-fronteras-abc2869ccab2.

8 En los siguientes enlaces se pueden observar dos ejemplos de páginas web como unidades didácticas: http://stikecriscr.wixsite.com/el-tablero-de-juego, http://alejandraalvaro528.wixsite.com/civilizaciones. 
permite concluir que las nuevas generaciones de docentes creen en la necesidad de incorporar paulatinamente la tecnología en los contextos de clase. Tomando en cuenta como se ha mencionado a lo largo del artículo que, la introducción de dichos enfoques, no necesariamente llevaría a una transformación completa de los problemas que enfrentan los contextos de aula en la actualidad, pero que si colaboran en la generación de nuevas prácticas didácticas que cambiarían sustancialmente el proceso de enseñanza-aprendizaje de la disciplina.

\section{Conclusiones}

Al inicio del taller, existe un escepticismo de parte del colectivo estudiantil. La costumbre focalizada en clases magistrales hace que se genere desconfianza hacia una propuesta innovadora desde la mediación y evaluación. Aunque la propuesta desarrollada en esta experiencia es amplia en el uso de herramientas y concepciones rupturistas desde el currículo y la mediación, se sabe que existen diferentes materiales y nuevas tecnologías que cotidianamente se pueden emplear. Pero quien agrega la pauta de la innovación educativa debe ser el colectivo docente, que se debe centralizar en su formación y estar actualizado de lo que sucede cotidianamente en su campo de trabajo.

Por otro lado, el profesorado universitario debe ser innovador. Su actualización es inminente en una época donde la tecnología educativa avanza considerablemente. Las clases universitarias no deben ser monótonas, sino propiciar aprendizajes que sean significativos. Las clases verticales donde el profesorado era el dueño absoluto del contenido no son funcionales en la actualidad, dado que a nivel social se esperan individuos que sean creativos y propositivos a las distintas realidades. Por lo tanto, la Universidad debe formar competencias aptas para enfrentar las diversas propuestas sociales sin dejar de lado el carisma humanista, el trabajo en equipo y la ética social.

Es necesario rescatar que la innovación educativa debe ser parte del profesorado en cualquier contexto profesional. Para innovar no es cuestión de edad, sino de poseer propuestas 
claras para cambiar el rumbo de la educación. Ahora bien, si desde los primeros niveles de formación académica se comienza con la motivación para realizar cambios en la labor docente, los resultados a futuro podrían ser más que positivos. Tomando en cuenta que la innovación educativa no solo se relaciona con el ámbito tecnológico, pero debe promover cambios significativos en la enseñanza y aprendizaje.

Desde los Estudios Sociales y la Educación Cívica queda mucho camino que recorrer. Se hace necesario rediseñar con clases magistrales que caracterizan parte del aprendizaje a nivel de secundaria. Dado que existe una evaluación en su mayoría sumativa que se convierte en un obstáculo cuando se quiere dar un salto cualitativo. Como consecuencia, no se generan aprendizajes significativos y se caracteriza la enseñanza de la historia como algo metódico y aburrido. Por tanto, se promueve un cambio desde la enseñanza universitaria, donde una adecuada mediación pueda ser aliciente para un aprendizaje significativo.

\section{Referencias}

Abarca, F. (2014). Implementación de estrategias de aprendizaje utilizando la red social de preferencia, como apoyo pedagógico para la enseñanza y el aprendizaje de los Estudios Sociales en los estudiantes de sétimo año del Liceo de Alajuelita, Circuito 06 de la Dirección Regional de Educación de San José Central (Tesis de licenciatura). Universidad Estatal a Distancia, San José, Costa Rica.

Alfaro, A. \& Navarro, R. (2016). Taller de producción Educativa para Estudios Sociales y Educación Cívica. Revista digital. Disponible en: https://issuu.com/rebeckanavarro/docs/tarea_1_curso_taller.docx_3a8ce7ab0f0fab.

Anaya, V., Ramos, L., Royo, J. \& Ureña, O. (2017). Taller de producción Educativa para Estudios Sociales y Educación Cívica. Padlet. Disponible en: https://padlet.com/josma9424/egg3tk7zlmwh.

Araya, A. \& Granados, F. (2017). Taller de producción Educativa para Estudios Sociales y Educación Cívica. Padlet. Disponible en: https://padlet.com/andf3n/d1lzj4sw9kc1.

Araya, A. \& Granados, F. (2017). Taller de producción Educativa para Estudios Sociales y Educación Cívica. E-portafolio. Disponible en: https://medium.com/@andrsarayavargas/aprobar-o-aprender-d6c00bb1c5ea. 
Araya, A. \& Granados, F. (2017). Taller de producción Educativa para Estudios Sociales y Educación Cívica. Video elaborado en el taller. Disponible en https://www.youtube.com/watch?time continue $=46 \& v=8 \mathrm{ZkHvVQvJ10}$.

Barrantes, R. (2014). Investigación: un camino al conocimiento. Un enfoque cuantitativo, cualitativo y mixto. San José, Costa Rica: EUNED.

Betanco, J., Fallas, A. \& Rodríguez, D. Taller de producción Educativa para Estudios Sociales y Educación Cívica. E-portafolio. Disponible en: https://medium.com/@jose1996bp/aprendizaje-sin-fronteras-abc2869ccab2.

Campos, J. \& Madriz, L. (2015). Investigación Acción en Contextos Educativos. San José, Costa Rica: EUNED.

Entrevista \#1(2017, 24 de setiembre). Entrevista con el estudiantado del Taller de Producción Educativa para Estudios Sociales y Educación Cívica del II ciclo 2017. Recuperado de: https://goo.gl/forms/81GVJ87Yhi3QDKq33.

Entrevista \#2 (2017, 1 de noviembre). Entrevista con el estudiantado del Taller de Producción Educativa para Estudios Sociales y Educación Cívica del II ciclo 2016. Recuperado de: https://goo.gl/forms/9WT0xa4mY4t0mRFP2.

Jiménez, F., Sánchez, M., Segura, K. \& Vargas, L. (2017). Taller de producción Educativa para Estudios Sociales y Educación Cívica. Padlet. Disponible en: 20 https://padlet.com/andresseg15/d895wggku34o.

Fallas, V. \& Trejos, I. (2013). Educación en la sociedad de la información y el conocimiento. San José, Costa Rica: EUNED.

Fernández, A. \& Murillo, M. (2016). Taller de producción Educativa para Estudios Sociales y Educación Cívica. Afiche digital. Disponible en: https://www.smore.com/91zj6-trabajocotidiano.

Fernández, A. \& Murillo, M. (2016). Taller de producción Educativa para Estudios Sociales y Educación Cívica. Página Web: Civilizaciones Antiguas. Disponible en: http://alejandraalvaro528.wixsite.com/civilizaciones.

Ferreiro, R. \& Vizoso, E. (2008). Una Condición Necesaria en el Empleo de las TI Cs en el Salón de Clases: La Mediación Pedagógica. En Revista Posgrado y Sociedad, (8) (2), $72-88$.

Hernández, R., Fernández, R. \& Baptista, P. (2010). Metodología de la investigación (5ª ed.). México D.F.: McGraw-Hill. 
Herrera, F. \& Murillo, M. (2016). Taller de producción Educativa para Estudios Sociales y Educación Cívica. Video elaborado en el taller. Disponible en https://www.youtube.com/watch?v=JdG5M-HrJMs\&feature=youtu.be.

Herrera, S., Montes, C., \& Ruíz, K. (2016). Taller de producción Educativa para Estudios Sociales y Educación Cívica. Los impactos de la globalización en América Latina. Disponible en: https://mx.ivoox.com/es/12494762.

Herrera, S., Montes, C., \& Ruíz, K. (2016). Taller de producción Educativa para Estudios Sociales y Educación Cívica. Página Web: El Tablero de Juego. Disponible en http://stikecriscr.wixsite.com/el-tablero-de-juego.

Jiménez, H. \& Mejías, L. (2016). Taller de producción Educativa para Estudios Sociales y Educación Cívica. Afiche digital. Disponible en: https://www.smore.com/5fz4k/.

Karagiorgi, Y. \& Symeou, L. (2005). Translating Constructivism into Instructional Design: Potential and Limitations. Educational Technology \& Society 8 (1), 17-27.

López, C., \& Heredia, Y. (2017). Marco de referencia para la evaluación de proyectos de innovación educativa - Guía de Aplicación. México, Nuevo León: Tecnológico de Monterrey.

López, M. \& Schmelkes, C. (2003). Definición del campo de investigación. En: López, A. (Ed.) Saberes científicos, humanísticos y tecnológicos: procesos de enseñanza y aprendizaje. Ciudad de México, México: Consejo Mexicano de Investigación Educativa.

Ramas, F. (Cord.).(2015). TIC en educación. Escenarios y experiencias. Ciudad de México, México: D.D.S.

Ruíz, K. (2016). Taller de producción Educativa para Estudios Sociales y Educación Cívica. Blog-portafolio: Alexandria Library. Disponible en http://alenxandrialibrary.blogspot.com/.

Serrano, R. \& Pontes, A. (2016). El desarrollo de la identidad profesional docente en la formación inicial del profesorado de secundaria. Enseñanza \& teaching, 34(1), 35-55. DOI: https://doi.org/10.14201/et20163413555.

Taracena, L. (2015). De la historia aburrida a la entretenida. Revista Perspectivas: Estudios Sociales y Educación Cívica, (10), 10-41. Disponible en: http://www.revistas.una.ac.cr/index.php/perspectivas/article/view/6749. 